\title{
Subject Index, Vol. 67, 1994
}

This index does not contain items ofthe reports ofthe Chromosome Mapping Workshops 1,2, 12, $15,22, \mathrm{X}$ and $\mathrm{Y}$ which are published in this volume.

Animals

Bubalus bubalus L. (river buffalo) 102

canine 81

Ctenomys flamarioni 193

Dama dama L. (fallow deer) 75

Drosophila 205

fish 31

Microtus kirgisorum (vole) 208

mouse $27,52,205$

pig $120,199,211$

rat $23,55,58$

sheep 114,137

Banded chromosome analysis Atlantic salmon (DAPI) 31 C. flamarioni (C, G, Ag-NOR) 193

canine (G) 81 fallow deer (RBA) 75 human (DAPI) 68 human (G) 41,116,190 human (Q) 37,190 human (R) 37,61,178, 187,205 M. kirgisorum (G) 208 mouse (Ag) 27 mouse (G) 27 mouse (QH) $27 \operatorname{pig}(\mathrm{G})$ 120,211 river buffalo $(\mathrm{G}, \mathrm{R}, \mathrm{Q}) 102$ sheep $(\mathrm{C}, \mathrm{G}, \mathrm{Q}) 137$

Chromosome aberration

bi-armed chromosomes in canine tumors 81 caused by gamma radiation 94 chromosome 9

variants 190 Ewing' s sarcoma translocation 129 Robertsonian translocations in sheep 137

segmental tetrasomy of mouse chromosome

$27 \mathrm{X} 0$ and $\mathrm{XX}$ mice 52

Chromosome mapping workshops chromosome 1 report 143 chromosome 2 report 215

chromosome 12 report 245 chromosome 15 report 1 chromosome 22 report $277 \mathrm{X}$ chromosome report 295 Y chromosome report 359

Comparative gene mapping Ctenomys 193 Drosophila 205 fish 31 mouse 205 pig 120,199,211

rat $23,55,58$

Fluorescent in situ hybridization (FISH) chromosome 9 variants 190 chromosome aberration by gamma radiation

94 Ewing's sarcoma translocation 129 gene mapping 23, 31, 37, 41, 61, 68, 69, 116,

$178,187,205,211$ genotyping errors in CEPH data 86 physical order by two-color FISH 86

telomeric fusions in canine tumors 81

Gene mapping Atlanta salmon

tandem repeats 31 human

Iq23 $\rightarrow \operatorname{lq} 2546$

$5 \mathrm{q} 3186$

ADRA1A, ADRA1B, ADRB2 69

ATP2B2, ATP2B3 41 
chromosome 16 cosmids 61

FLU 129

FY 68

HH 126

LCK 187

MEGE1 116

PITPN 205

SCN1A 178 mouse

Pitpn 205 Pig

CRC 211

GH 211

GPI 211

LIPE 211

microsatellites 199

NGFB 120 rat

Calnal, Calna2 55

N-ras 23

Ppplcc 58

Heterochromatin Atlanta salmon 31 C. flamarioni 193 chromosome 9 variants 190 sheep 137

Human disorders

hereditary hemochromatosis (HH) 126 melanoma 116

primitive neuroectodermal tumors 129 Turner syndrome and RPS4X 52

Hybrids

human-rodent 46,116,178 radiation hybrids 61,69 rat-mouse 55, 58

Karyotype canine 81 fallow deer 75 M. kirgisorum 208 river buffalo 102 sheep 114

Linkage

HH locus 126

human $5 \mathrm{q} 3186$

porcine chromosome 1199

Obituary

S.P. Ray-Chaudhuri 141

Physical mapping

13q 12 containing FLT1 and FLT3 175 250kbonllq24 129

Repetitive sequence Atlanta salmon 31 human chromosome 1661 satellite probes 190

Ribosomal RNA gene (rDNA) Atlanta salmon 31

Sequence analysis

Atlantic salmon 5 S rDNA 31 pig NGFB 120 SCN1A 178

Viral integration site

Adenovirus 5/SV40 37

Techniques

direct in situ single copy (DISC)-PCR 199 effect of chromosome size and aberration by

gamma radiation 94 genomic difference cloning 46

$\mathrm{X}$-chromosome inactivation

Rps4 mRNA in X0 and XX mice 52 
Cytogenet Cell Genet, Vol. 67,1994 